\title{
Drug diffusion from polymeric delivery devices: a problem with two moving boundaries
}

\author{
Mike Hsieh $^{1} \quad$ Scott W. McCue ${ }^{2}$ \\ Timothy J. Moroney ${ }^{3}$ \\ Mark I. Nelson ${ }^{4}$
}

(Received 29 January 2011; revised 4 July 2011)

\begin{abstract}
An existing model for solvent penetration and drug release from a spherically shaped polymeric drug delivery device is revisited. The model has two moving boundaries, one that describes the interface between the glassy and rubbery states of the polymer, and another that defines the interface between the polymer ball and the pool of solvent. The model is extended so that the nonlinear diffusion coefficient of drug explicitly depends on the concentration of solvent, and the resulting equations are solved numerically using a front fixing transformation together with a finite difference spatial discretisation and the method of lines. We present evidence that our scheme is much more accurate than a previous scheme. Asymptotic results in the small time limit are presented, which show how the use of a kinetic law as a boundary condition on the innermost moving boundary dictates qualitative behaviour, the scalings being very different to the similar
\end{abstract}

http://anziamj. austms.org.au/ojs/index.php/ANZIAMJ/article/view/3940 gives this article, (c) Austral. Mathematical Soc. 2011. Published August 3, 2011. ISSN 1446-8735. (Print two pages per sheet of paper.) Copies of this article must not be made otherwise available on the internet; instead link directly to this URL for this article. 
moving boundary problem that arises from modelling the melting of an ice ball. The implication is that the model considered here exhibits what is referred to as non-Fickian or Case II diffusion which, together with the initially constant rate of drug release, has certain appeal from a pharmaceutical perspective.

\section{Contents}

1 Introduction

C550

2 Numerical method

C554

3 Small time analysis

C556

4 Numerical results

C558

5 Conclusion

C563

References

C563

\section{Introduction}

The pharmaceutical industry extensively makes use of polymeric materials to construct controlled drug release devices. Of interest here are hydrophilic polymers (for example, certain hydrogels) that are characterised by swelling and enhanced drug diffusion when in contact with a thermodynamically compatible solvent. In particular, we are concerned with polymers that exist in a hard glassy state when they are stored, during which time a particular drug is distributed evenly throughout (such a drug may be dissolved in the polymer when it is manufactured). Furthermore, when such a polymer is placed in an aqueous environment the solvent starts to penetrate into the 
polymer and, as a result of the subsequent disentanglement of the affected macromolecular polymer chains, the hard glassy state transforms into a soft rubbery state. There is an increased volume of polymer in the rubbery state and, importantly, the drug mobility in the rubbery state is significantly higher than in the glassy state. We are not concerned with a particular polymer, drug or solvent with the above properties; however, the parameter values used in this paper are taken from studies $[1,8,10,11]$ that involve the polymers polyvinyl alcohol (PVA) and hydroxypropyl methylcellulose (HPMC) and the solvents water, phosphate buffer and hydrogen chloride. The drugs used in these particular studies include chlorpheniramine maleate, theophylline, buflomedil pyridoxal phosphate and sodium diclofenac.

Let the concentration of solvent in a polymer ball be denoted by $U(R, T)$ and the concentration of drug by $V(R, T)$, where $R$ is the radial distance and $\mathrm{T}$ is time. The problem we are concerned with in the present study was proposed by Cohen and Erneux [3] in one spatial dimension and then treated by Lin and Peng [6] for the spherical geometry of interest here, except that we extend the model to include nonlinear diffusion. The model is roughly a combination of Higuchi's model [4] to describe the transport of the drug and a moving boundary problem for the penetration of solvent as described by Astarita and Sarti [1]. There are two moving boundaries in the problem, with locations denoted by $R=S_{1}(T)$ and $R=S_{2}(T)$; the first is the interface between the rubbery and glassy regions in the polymer while the second is the outer boundary of the polymer ball. The rubbery-glassy interface $R=S_{1}(T)$ moves towards the centre of the sphere as the solvent penetrates the polymer, while the outer boundary $R=S_{2}(T)$ moves outwards as the polymer swells. Whereas we assume that no drug is released from the inner glassy core of the polymer ball, there is diffusion of drug from the outer rubbery shell $S_{1}(T)<R<S_{2}(T)$ with a diffusion coefficient that is an increasing function of the solvent concentration $\mathrm{U}$.

We scale the problem with the dimensionless variables

$$
r=\frac{R}{S_{1}(0)}, \quad t=\frac{D_{s} T}{S_{1}^{2}(0)}, \quad v=\frac{V}{V_{\text {init }}}, \quad u=\frac{U-U^{*}}{U_{0}-U^{*}}, \quad s_{i}(t)=\frac{S_{i}(T)}{S_{1}(0)},
$$


where $D_{s}$ is the diffusivity of the solvent, $V_{\text {init }}$ is the initial concentration of drug in the polymer, $\mathrm{U}_{0}$ is the equilibrium solvent concentration at the surface of the spherical pellet, $\mathrm{U}^{*}$ is the threshold value of the solvent concentration to transform the polymer from the glassy state to the rubbery state, and $i$ takes the values 1 or 2 . As a result, the dimensionless problem for the diffusion of solvent in the rubbery region is

$$
\begin{array}{ll}
\frac{\partial u}{\partial t}=\frac{1}{r^{2}} \frac{\partial}{\partial r}\left(r^{2} \frac{\partial u}{\partial r}\right) & \text { in } s_{1}(t)<r<s_{2}(t), \\
u=1 & \text { at } r=s_{2}(t), \\
\frac{\partial u}{\partial r}=-(u+\lambda) \frac{d s_{1}}{d t} & \text { at } r=s_{1}(t), \\
u^{n}=-\mu \frac{d s_{1}}{d t} & \text { at } r=s_{1}(t),
\end{array}
$$

with

$$
s_{2}^{3}(t)-1=3 v_{m} \int_{s_{1}(t)}^{s_{2}(t)}\left[1-\frac{1-u}{\lambda+1}\right] r^{2} d r .
$$

Physically, (1) describes linear diffusion of solvent in the rubbery region $s_{1}(t)<r<s_{2}(t)$, while (2) states that the concentration of solvent on the outer boundary is equal to the concentration in the body of solvent surrounding the polymer ball (which, in dimensional variables, is the equilibrium value $\mathrm{U}_{0}$ ). The moving boundary condition (3) is a mass balance, while (4) describes a kinetic law relating the speed of the rubbery-glassy interface to the level of solvent concentration above the threshold value $\mathrm{U}^{*}$. The integral relationship (5) is another mass balance that relates the volume increase to the molar volume of the solvent.

The dimensionless problem for the diffusion of the drug is

$$
\begin{array}{ll}
\frac{\partial v}{\partial t}=\frac{1}{r^{2}} \frac{\partial}{\partial r}\left(r^{2} D(u) \frac{\partial v}{\partial r}\right) & \text { in } s_{1}(t)<r<s_{2}(t), \\
v=0 & \text { at } r=s_{2}(t),
\end{array}
$$




$$
\mathrm{D}(\mathrm{u}) \frac{\partial v}{\partial \mathrm{r}}=(1-v) \frac{\mathrm{d} \mathrm{s}_{1}}{\mathrm{dt}} \quad \text { at } r=s_{1}(\mathrm{t}),
$$

where the nonlinear diffusion coefficient is

$$
\mathrm{D}(\mathrm{u})=\delta \mathrm{e}^{-\beta(1-\mathrm{u})} .
$$

Once the rubbery-glassy interface $r=s_{1}(t)$ reaches the centre of the ball, the drug release continues with (3)-(4) replaced with $\partial u / \partial r=0$ on $r=0$ and (8) replaced with $\partial v / \partial r=0$ on $r=0$. Physically, (6) is a diffusion equation for the drug with a nonlinear diffusion coefficient $D(u)$ that is an increasing function of the concentration of solvent. The drug concentration is assumed to vanish on the surface of polymer ball, leading to (7). Finally, (8) describes the mass balance of drug at the rubbery-glassy interface.

There are six dimensionless parameters in (1)-(9): $\delta=\mathrm{D}_{\mathrm{d}} / \mathrm{D}_{\mathrm{s}}$, the ratio of the diffusion coefficient of drug at the outer boundary to the (constant) diffusion coefficient of solvent; $\beta \geqslant 0$, a measure of the nonlinearity in the dependence of diffusivity of drug on concentration of solvent; $0 \leqslant v_{m}<1$, the product of $\mathbf{U}_{0}$ with the molar volume of solvent when it has penetrated into the polymer; $\lambda=\mathbf{U}^{*} /\left(\mathbf{U}_{0}-\mathbf{U}^{*}\right)$, a control parameter that increases as the difference between the equilibrium value and the threshold value of the solvent concentration decreases; $\mu=\mathrm{Dk}_{1}^{-1}\left(\mathrm{U}_{0}-\mathrm{U}^{*}\right)^{-\mathrm{n}} / \mathrm{S}_{2}(0)$, a kinetic parameter that is determined by fitting the empirical law (4) to experiments; and $\mathfrak{n}$, an exponent also found by the same experimental test.

In this model the processes of solvent and drug diffusion are only coupled in one direction, in the sense that the double moving boundary problem $(1)-(5)$ is solved for $u(r, t), s_{1}(t)$ and $s_{2}(t)$ without reference to (6)-(9); however (6)(9) requires the solutions for $u(r, t), s_{1}(t)$ and $s_{2}(t)$ as inputs. The problem $(1)$ (5) also describes a one phase Stefan problem for melting an ice ball, with the unusual boundary condition (4) modelling the effect of kinetic undercooling on melting temperature (with $\mu=0$, the problem (1)-(5) resembles a more classical Stefan problem with a constant melting temperature).

As mentioned above, (1)-(9) with $\beta=0$ was treated by Lin and Peng [6]; the problem with $v_{\mathrm{m}}=0$ (no swelling) was also studied recently by McCue 
et al. [7]. We revisit this problem and Section 2 generates numerical solutions using a front fixing transformation and finite differences. We argue that our method is much more accurate than that used by Lin and Peng [6] and point out some instances in which they display solutions that are not physically realistic. Section 3 summarises a small time analysis that demonstrates the role of the kinetic parameter $\mu$ in determining the speed of the moving boundaries and the early rate of drug release. Some further numerical results are discussed in Section 4.

\section{Numerical method}

The model of the swelling controlled release system is a non-linear moving boundary problem with two moving boundaries, and is more difficult to treat numerically than standard linear diffusion problems. The method we use to solve this problem numerically is to first transform the moving boundary problem into a fixed boundary problem via a standard 'front fixing' method which maintains the nature of the original moving boundary problem, but results in an extra term appearing in the transformed governing equation. This extra term is of convection type with no physical significance to the swelling controlled release system.

By applying the front fixing transformation

$$
\xi=\frac{r-s_{1}}{s_{2}-s_{1}}
$$

to the problem (1)-(9) we obtain

$$
\begin{aligned}
\left(s_{2}-s_{1}\right)^{2} \frac{\partial v}{\partial t}= & D(u)\left\{\frac{\partial^{2} v}{\partial \xi^{2}}+\frac{2\left(s_{2}-s_{1}\right)}{\left[s_{1}+\left(s_{2}-s_{1}\right) \xi\right]} \frac{\partial v}{\partial \xi}\right\}+\beta D(u) \frac{\partial u}{\partial \xi} \frac{\partial v}{\partial \xi} \\
& +\left(s_{2}-s_{1}\right)\left[(1-\xi) \frac{d s_{1}}{d t}+\xi \frac{d s_{2}}{d t}\right] \frac{\partial v}{\partial \xi} \quad \text { in } 0<\xi<1, \\
v= & \text { at } \xi=1,
\end{aligned}
$$




$$
\begin{aligned}
D(u) \frac{\partial v}{\partial \xi}= & (1-v)\left(s_{2}-s_{1}\right) \frac{d s_{1}}{d t} \quad \text { at } \xi=0, \\
\left(s_{2}-s_{1}\right)^{2} \frac{\partial u}{\partial t}= & \frac{\partial^{2} u}{\partial \xi^{2}}+\frac{2\left(s_{2}-s_{1}\right)}{\left[s_{1}+\left(s_{2}-s_{1}\right) \xi\right]} \frac{\partial u}{\partial \xi} \\
& +\left(s_{2}-s_{1}\right)\left[(1-\xi) \frac{d s_{1}}{d t}+\xi \frac{d s_{2}}{d t}\right] \frac{\partial u}{\partial \xi} \quad \text { in } 0<\xi<1, \\
u & =1 \quad \text { at } \xi=1, \\
\frac{\partial u}{\partial \xi}= & -(u+\lambda)\left(s_{2}-s_{1}\right) \frac{d s_{1}}{d t} \quad \text { at } \xi=0, \\
u^{n}= & -\mu \frac{d s_{1}}{d t} \quad \text { at } \xi=0,
\end{aligned}
$$

with

$$
s_{2}^{3}=1+v_{m} \frac{\lambda}{\lambda+1}\left(s_{2}^{3}-s_{1}^{3}\right)+\frac{3 v_{m}}{\lambda+1} \int_{0}^{1} u\left[s_{1}+\left(s_{2}-s_{1}\right) \xi\right]^{2}\left(s_{2}-s_{1}\right) d \xi .
$$

The idea behind using (10) is that (11)-(18) now applies on the fixed domain $0 \leqslant \xi \leqslant 1$, with $\xi=0$ and $\xi=1$ corresponding to $r=s_{1}(t)$ and $r=s_{2}(t)$, respectively.

The numerical method we use to address the problem (11)-(18) is the Method of Lines with finite difference spatial discretisation. The governing equations (11) and (14) are discretised in space by using the finite difference method to form ordinary differential equations (ODEs) at each internal node and the boundary node $\xi=0$. The solutions for drug and solvent concentration at the boundary node $\xi=1$ are known due to the corresponding Dirichlet conditions there. In particular, we utilise the second order central difference formulae

$$
\left.\frac{\partial u}{\partial \xi}\right|_{\xi=\xi_{i}} \approx \frac{u_{i+1}-u_{i-1}}{2 \Delta \xi},\left.\quad \frac{\partial^{2} u}{\partial \xi^{2}}\right|_{\xi=\xi_{i}} \approx \frac{u_{i+1}-2 u_{i}+u_{i-1}}{(\Delta \xi)^{2}},
$$

except at the boundary node $\xi=0$ where we employ the first order forward difference formula and the second order central difference formula with a 
ghost node. The resultant system of ODEs is solved numerically with the Matlab built-in ODE/DAEs solver ode15i, which solves fully implicit ODEs by employing variable order and variable stepsize numerical differentiation formulae. The solver chooses both the order of the scheme and time stepsize adaptively to meet local error tolerances, so we are unable to specify the exact time steps used for each run. Further, it is necessary to use the small time solution described in Section 3 as an initial condition for the scheme, as the inner and outer moving boundaries coincide at $t=0$.

The last issue relates to the integral in (18) which is subtle and not straightforward to discretise by the finite difference spatial discretisation. There are two approaches to form a differential algebraic equation for (18). One approach utilises existing formulae for numerical integration. The other approach, which we employ, is to derive the explicit form of the speed of the volume expansion front $s_{2}(t)$ based on the idea used by Radu et al. [9]. We differentiate both sides of equation (5) and apply the Leibniz integral rule for differentiating a definite integral whose limits are a function of the differential variable. We then transform the independent variable $r$ to the new independent variable $\xi$ given by the front fixing transformation (10). As a result, we find that (18) becomes

$$
\frac{\mathrm{d} s_{2}}{\mathrm{dt}}=\left.\frac{1}{s_{2}-s_{1}} \frac{v_{\mathrm{m}}}{1-v_{\mathrm{m}}} \frac{1}{\lambda+1} \frac{\partial u}{\partial \xi}\right|_{\xi=1},
$$

which is discretised using a backward difference formula.

\section{Small time analysis}

We investigate the small time behaviour of the problem (1)-(9) by applying the following expansions:

$$
u \sim \frac{1}{r}\left\{u_{0}(\xi)+u_{1}(\xi)\left(1-s_{1}\right)+u_{2}(\xi)\left(1-s_{1}\right)^{2}\right\},
$$




$$
\begin{aligned}
v & \sim \frac{1}{r}\left\{v_{0}(\xi)+v_{1}(\xi)\left(1-s_{1}\right)+v_{2}(\xi)\left(1-s_{1}\right)^{2}\right\} \\
\frac{d s_{1}}{d t} & \sim g_{0}+g_{1}\left(1-s_{1}\right)+g_{2}\left(1-s_{1}\right)^{2} \\
s_{2} & \sim 1+h_{1}\left(1-s_{1}\right)+h_{2}\left(1-s_{1}\right)^{2},
\end{aligned}
$$

as $s_{1} \rightarrow 1^{-}$to $(1)-(9)$, where $\xi$ is given by (10). The ansatz (19)-(22) is anticipated by adapting previous studies [1, 2, 7] to allow for the second moving boundary and the presence of drug. Substituting (19)-(22) into (1)(9) provides differential equations for the $\boldsymbol{u}_{i}$ and $\boldsymbol{v}_{\boldsymbol{i}}$ which are solved to give (in original variables $r$ and $t$ )

$$
\begin{aligned}
& u \sim 1-\frac{1+\lambda}{\mu}\left(\frac{1-r}{r}\right)-\frac{v_{m}(1+\lambda)}{2 \mu^{2}\left(1-v_{m}\right)} \frac{(1-r)^{2}}{r}, \\
& v \sim \frac{1-r}{\delta \mu r}\left(1+\frac{v_{m}(1-r)}{2 \delta \mu\left(1-v_{m}\right)}+\frac{\beta(1+\lambda)(1-r)}{2 \mu}\right), \\
& s_{2} \sim 1+\frac{v_{m}}{1-v_{m}} \frac{t}{\mu}-\frac{v_{m}\left\{1+[n(1+\lambda)+2 \mu]\left(1-v_{m}\right)\right\} t^{2}}{2 \mu^{3}\left(1-v_{m}\right)^{3}}, \\
& s_{1} \sim 1-\frac{t}{\mu}+\frac{n(1+\lambda)}{2 \mu^{3}\left(1-v_{m}\right)} t^{2},
\end{aligned}
$$

as $\mathbf{t} \rightarrow \mathbf{O}^{+}$. Terms $\mathcal{O}(\mathbf{t})$ in $(23)-(24)$ and $\mathcal{O}\left(\mathbf{t}^{3}\right)$ in (25)-(26) were also calculated, but these are quite lengthy and we omit them for brevity.

It is of interest to compute the amount of drug released from the polymer sphere at time $\mathrm{t}$ :

$$
m_{t}=-\left.3 \delta \int_{0}^{t} s_{2}^{2} \frac{\partial v}{\partial r}\right|_{r=s_{2}} d t .
$$

This quantity is simply the integral of the outward flux of drug evaluated at the outer boundary $r=s_{2}(t)$ and normalised so that $m_{t} \rightarrow 1^{-}$as $t \rightarrow \infty$. In other words, $m_{t}$ is the ratio of the drug released at time $t$ to the amount of drug initially dissolved in the polymer. Using our results above (and the 
next order terms not included here), we find that

$$
m_{t} \sim \frac{3 t}{\mu}-\frac{3\left[1+\delta n(1+\lambda)\left(1-v_{m}\right)+2 \delta \mu\left(1-v_{m}\right)^{2}\right] t^{2}}{2 \delta \mu^{3}\left(1-v_{m}\right)^{2}} \quad \text { as } t \rightarrow 0^{+} \text {. }
$$

The above results are of considerable interest for a number of reasons. First, the interface speeds $d s_{1} / d t$ and $d s_{2} / d t$ are both $\mathcal{O}(1)$ in the limit $t \rightarrow 0^{+}$ which, together with $\partial u / \partial r\left(s_{2}, t\right)=\mathcal{O}(1)$, is unusual for moving boundary problems of this sort (this phenomenon is often referred to as non-Fickian or Case II diffusion). Indeed, for the case $\mu=0$, the system (1)-(5) resembles a Stefan problem for melting a ice ball, and the interface speeds are both $\mathcal{O}\left(t^{-1 / 2}\right)$ in the limit, the more common scaling. Thus we see that the kinetic boundary condition (4) with $\mu>0$ is critical in determining qualitative behaviour, at least for small time. Further, the problem with $\mu>0$ leads to $m_{t}=\mathcal{O}(t)$ which corresponds to a constant rate of drug release for early times, a design output that is often desirable in the pharmaceutical industry. For $v_{m}=0$ there is no swelling, and the above results reduce to those given by McCue et al. [7], where the $v_{m}=0$ problem was considered.

\section{Numerical results}

Representative numerical results are presented in Figures 1 and 2, computed using 3001 spatial grid points. For these two figures the parameter values were chosen by taking typical dimensional quantities from the literature $[8,10,12]$ and rescaling appropriately. We see that in this case the glassy-rubbery interface $r=s_{1}(t)$ reaches the centre of the polymer ball at $t_{e}=0.4606$, at which time the outer boundary $s_{2}=1.5462$ and the polymer ball has swollen to 3.70 times its initial volume. The profiles of solvent and drug concentration at $t_{e}=0.4606$ are indicated by the blue solid curves. As mentioned above, after this time the boundary conditions at $r=s_{1}(t)$ are replaced by no flux conditions at $r=0$. The swelling continues and we find that $s_{2} \rightarrow 1.6388$ for 

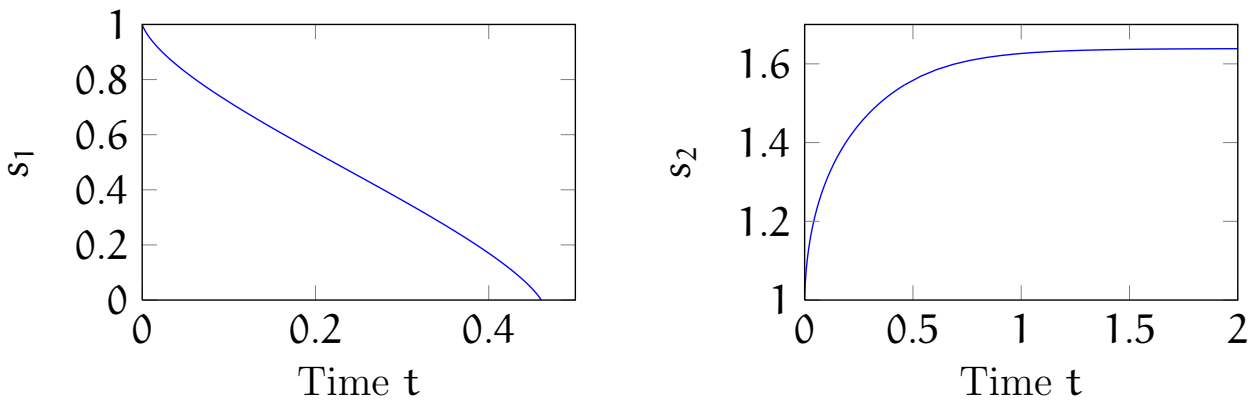

Figure 1: Numerical results for the position of the moving boundaries $r=s_{1}(t)$ and $r=s_{2}(t)$ for parameter values $\delta=0.1, \beta=5.55, v_{m}=0.77$, $\lambda=0.8, \mu=0.1$ and $n=1$.

large times, implying that the sphere swells to 4.40 times its initial volume for these parameter values.

Recall the model (1)-(9) with $\beta=0$ was studied by Lin and Peng [6] (our model extends Lin and Peng [6] by permitting $\beta>0$ ). They applied a numerical scheme that involves an approximate similarity solution on each time step that unfortunately becomes less accurate as time progresses. As a consequence, their results for moderate to large times are unreliable. For example, Figure 3 plots the outward flux of drug at $r=s_{2}(t)$ for a variety of parameter values. The parameter values and scalings on each axis are the same as those used by Lin and Peng [6, Figures 13 and 14]. In both parts (a) and (b), the blue curves are taken by scanning the figures shown by Lin and Peng [6] and digitising the data, while the red curves are our numerical results. The results obtained by Lin and Peng [6] all have a distinct local minimum followed by a sharp rise, whereas our results are monotonically decreasing, which is expected as the flux $\partial v / \partial r \rightarrow 0$ as $t \rightarrow \infty$ for all $r$.

Further, consider the parameter $v_{\mathfrak{m}}$, which is defined to be the additional volume that the polymer occupies when one mole of the solvent has penetrated into the polymer ball divided by the volume the mole occupies in the solvent 


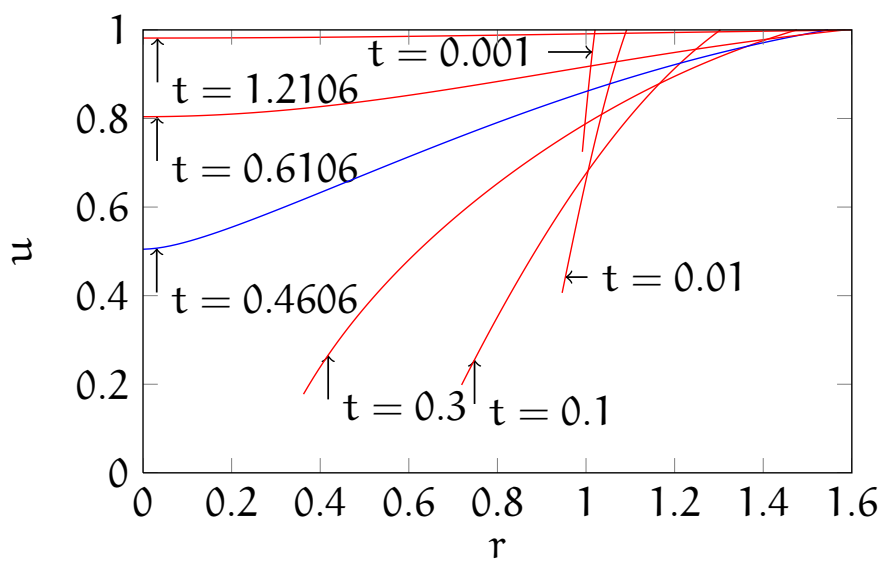

(a) profiles of solvent concentration for times $t=0.001,0.01$, $0.1,0.3,0.4606$ (blue), 0.6106 and 1.2106 .

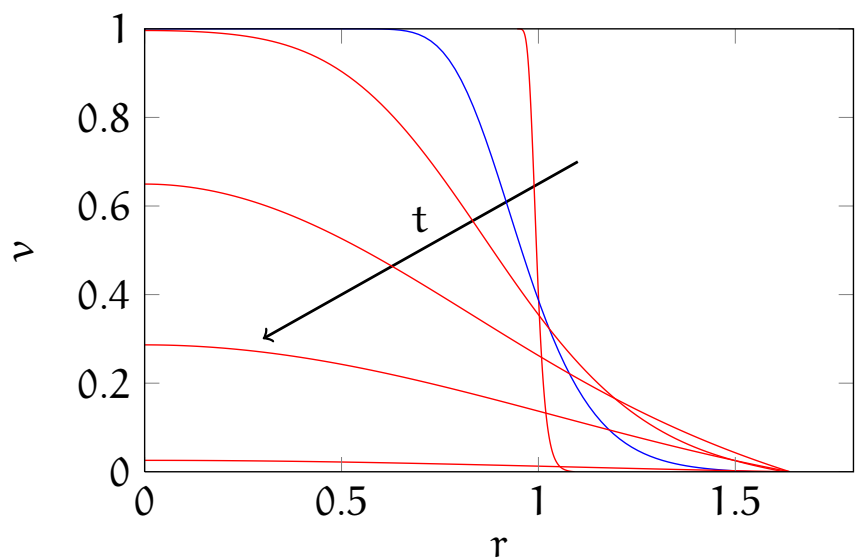

(b) profiles of drug concentration for times $t=0.01$, 0.4606 (blue), $0.9606,2.0606,3.9606$ and 10.4606. The arrow in part (b) indicates increasing time.

Figure 2: Numerical results for the same parameter values as in Figure 1. 


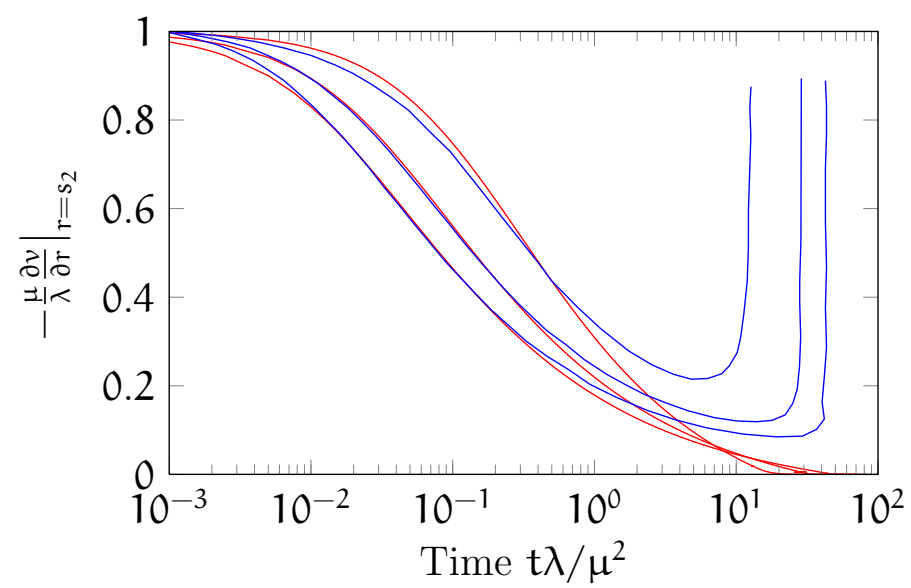

(a) $\beta=0, \delta=1, v_{m}=0.116, \lambda=1$ and $\mu=0.2$, with profiles from top to bottom drawn for $n=1,5$ and 10 .

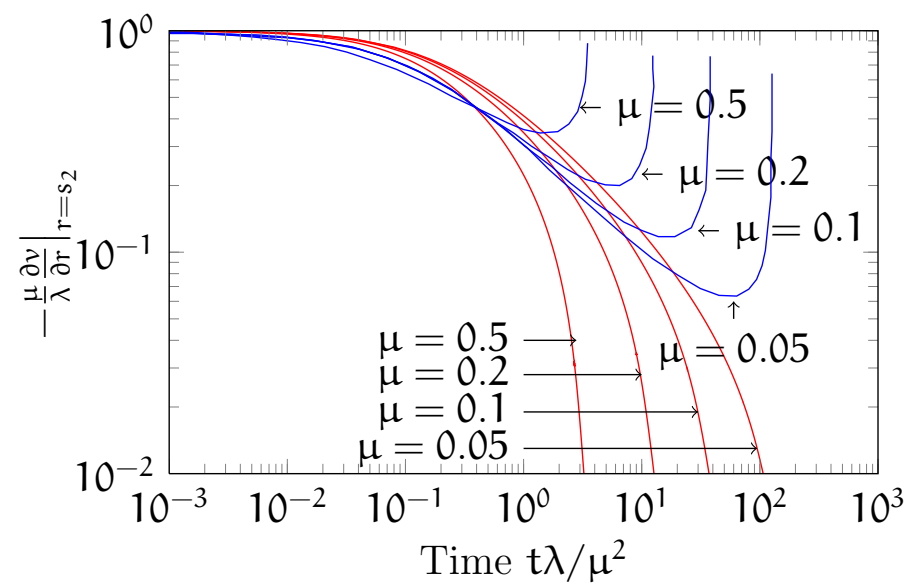

(b) $\beta=0, \delta=1, v_{m}=0.116, \lambda=1$ and $n=1$, with profiles for $\mu=0.5,0.2,0.1$ and 0.05 .

Figure 3: Comparison of our numerical results with Lin and Peng [6] for the outward flux of drug at the surface of the spherical polymer versus time (both the flux and time are scaled in the same way). The blue solid lines are results obtained by Lin and Peng [6] and the red solid lines are results obtained numerically by solving (1)-(9) with 3001 spatial grid points. 

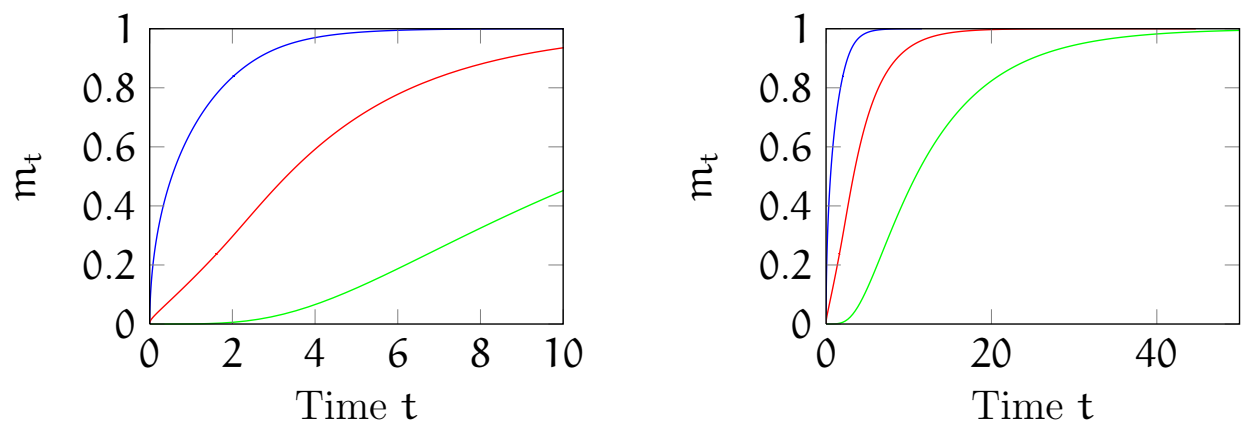

Figure 4: Normalised drug release from the swelling polymer versus time for $\delta=0.1, n=1, \beta=0, \mu=0.1$ and for $\lambda=0.2$ (green), 5 (red) and 10 (blue).

pool that surrounds the polymer. Lin and Peng [6] present results (in their dimensionless variables) that are for $v_{m}>1$, which is not a physically realistic regime, unless there is perhaps some additional chemistry that causes the polymer to swell by more than the volume of solvent diffusing into it. This would lead to alterations in other aspects of the model. As it stands, our numerical scheme works only for $0 \leqslant v_{m}<1$. Furthermore, the small time results presented in Section 3 break down as $v_{m} \rightarrow 1^{-}$, as do the large $\lambda$ asymptotics outlined by Lin and Peng [6].

One particular issue in designing swelling controlled release systems is the need to vary the rate of drug release. To take one simple example, Figure 4 presents the dependence of the normalised drug release $\boldsymbol{m}_{\mathrm{t}}$ (computed using (27)) on time for a fix set of parameters except the control parameter $\lambda$, for which we chose $\lambda=0.2,5$ and 10 . We see that changing this parameter has a significant effect on drug release. For example, at dimensionless time $t=4$ almost all the drug is released for $\lambda=0.2$, but very little is released for $\lambda=10$. A thorough investigation of the parameter space is likely to reveal a number of strategies for controlling drug release. 


\section{Conclusion}

This study concerns the problem (1)-(9), a moving boundary problem with two moving boundaries. This problem with $\beta=0$ was treated by Lin and Peng [6], and is based on a one dimensional version proposed by Cohen and Erneux [3]. The model couples together the processes of solvent penetration and drug diffusion from a polymeric drug delivery device which is initially in a glassy state and transitions to a rubbery state after contact with the solvent. Our problem (1)-(9) with $\beta>0$ is an extension of that treated by Lin and Peng [6], in that we included a nonlinear diffusion coefficient for drug diffusion that increases with solvent concentration.

Our main results are twofold. First, we present a numerical scheme that appears to be considerably more accurate than that described by Lin and Peng [6]. Second, we provide new asymptotic results for the small time limit that explicitly show the unusual scalings that result from including the kinetic boundary condition (4) in the model. In particular, the parameter $\mu>0$ (which denotes kinetic undercooling in the corresponding Stefan problem for a melting ice ball) acts to regularise singular behaviour in the limit $t \rightarrow 0^{+}$, leading to physically more meaningful results. Finally, due to space restrictions we were unable to explore the role that each parameter has on the solutions of the problem (1)-(9), nor the consequence in terms of designing drug release devices. This exercise, as well as including further effects such as polymer dissolution, is left for further study.

\section{References}

[1] G. Astarita and G. C. Sarti. A class of mathematical models for sorption of swelling solvents in glassy polymers. Polymer Engineering and Science., 18, 1978, 388-395. doi:10.1002/pen.760180510. C551, C557 
[2] D. S. Cohen and T. Erneux. Free boundary problems in controlled release pharmaceuticals. I: Diffusion in glassy polymers. SIAM Journal on Applied Mathematics., 48, 1988, 1451-1465. http://www. jstor.org/stable/2101759. C557

[3] D. S. Cohen and T. Erneux.. Free boundary problems in controlled release pharmaceuticals. II: Swelling-controlled release. SIAM Journal on Applied Mathematics., 48, 1988, 1466-1474. http://www.jstor.org/stable/2101760. C551, C563

[4] T. Higuchi. Mechanism of sustained-action medication: theoretical analysis of rate of release of solid drugs dispersed in solid matrices. Journal of Pharmaceutical Sciences., 52, 1963, 1145-1149. doi:10.1002/jps.2600521210. C551

[5] J.-S. Lin, C.-C. Hwang, C.-M. Lin, and J.-Y. Lai. Solvent transport in spherical polymer-penetrant systems. Chemical Engineering Science., 56, 2001, 151-156. doi:10.1016/S0009-2509(00)00410-3.

[6] J.-S. Lin and Y.-L. Peng. Swelling controlled release of drug in spherical polymer-penetrant systems. International Journal of Heat and Mass Transfer., 48, 2005, 1186-1194.

doi:10.1016/j.ijheatmasstransfer.2004.08.031. C551, C553, C554, C559, C561, C562, C563

[7] S. W. McCue, M. Hsieh, T. J. Moroney, and M. I. Nelson. Asymptotic and numerical results for a model of solvent-dependent drug diffusion through polymeric spheres. Submitted. C554, C557, C558

[8] B. Narasimhan and N. A. Peppas. Molecular analysis of drug delivery systems controlled by dissolution of the polymer carrier. Journal of Pharmaceutical Sciences., 86, 1997, 297-304. doi:10.1021/js960372z. C551, C558

[9] F. A. Radu, M. Bause, P. Knabner, G. W. Lee, and W. C. Friess. Modeling of drug release from collagen matrices. Journal of 
Pharmaceutical Sciences, 91, 2002, 964-972. doi:10.1002/jps.10098. C556

[10] J. Siepmann, K. Podual, M. Sriwongjanya, N. A. Peppas and R. Bodmeier. A new model describing the swelling and drug release kinetics from hydroxypropyl methylcellulose tablets. Journal of Pharmaceutical Sciences., 88, 1999, 65-72. doi:10.1021/js9802291. C551, C558

[11] S. Kill and K. Dam-Johansen. Controlled drug delivery from swellable hydroxypropylmethylcellulose matrices: model-based analysis of observed radial front movements. Journal of Controlled Release., 90, 2003, 1-21. doi:10.1016/S0168-3659(03)00122-6. C551

[12] N. Wu, L.-S. Wang, D. C.-W. Tan, S. M. Moochhala, and Y.-Y. Yang. Mathematical modeling and in vitro study of controlled drug release via a highly swellable and dissoluble polymer matrix: polyethylene oxide with high molecular weights. Journal of Controlled Release., 102, 2005, 569-581. doi:10.1016/j.jconrel.2004.11.002. C558

\section{Author addresses}

1. Mike Hsieh, Mathematical Sciences, Queensland University of Technology, Brisbane, Queensland 4001, Australia. mailto:m2.hsieh@student.qut. edu.au

2. Scott W. McCue, Mathematical Sciences, Queensland University of Technology, Brisbane, Queensland 4001, Australia. mailto:scott.mccue@qut.edu.au

3. Timothy J. Moroney, Mathematical Sciences, Queensland University of Technology, Brisbane, Queensland 4001, Australia. mailto:t.moroney@qut. edu. au

4. Mark I. Nelson, School of Mathematics and Applied Statistics, University of Wollongong, Wollongong, New South Wales 2522, 
Australia.

mailto:mnelson@uow.edu. au 